Bundesgesundheitsbl $2013 \cdot 56: 1418-1424$

DOI 10.1007/s00103-013-1832-0

Online publiziert: 5. September 2013

(c) Springer-Verlag Berlin Heidelberg 2013

F. Giesel ${ }^{1} \cdot$ K. Köhler ${ }^{1}$ E. Nowossadeck ${ }^{2}$

${ }^{1}$ Institut für Verkehrsforschung, Deutsches Zentrum für Luft- und Raumfahrt e.V. (DLR), Berlin

${ }^{2}$ Robert Koch-Institut, Berlin

\title{
Alt und immobil auf dem Land?
}

\section{Mobilitätseinschränkungen älterer Menschen vor dem Hintergrund einer zunehmend problematischen Gesundheitsversorgung in ländlichen Regionen}

Ein prägendes Merkmal ländlicher Regionen ist die geringe Bevölkerungsdichte (gemessen beispielsweise als Einwohner pro Quadratkilometer). Zudem gibt es in diesen Regionen relativ wenige Städte und vor allem Großstädte. Jedoch ist in diesen ein Großteil der öffentlichen Daseinsvorsorge angesiedelt. Im Vergleich zu städtischen Räumen sind ländliche Regionen verstärkt von Erreichbarkeitsproblemen betroffen: Aktuelle Befunde bestätigen am Beispiel von Einkaufsgelegenheiten, dass sich deren Erreichbarkeit mit abnehmender Gemeindegröße schwieriger gestaltet. Diese Situation wird infolge sozialer Benachteiligung verschärft, die in ländlichen Regionen ebenfalls weiter verbreitet ist [1].

Vor dem Hintergrund des demografischen Wandels ist zudem die Erreichbarkeit von Infrastrukturen der Gesundheitsversorgung von zentraler Bedeutung. Die Verfügbarkeit von Krankenhäusern und Fachärzten hängt mit der Bevölkerungsdichte zusammen, jedoch orientiert sich die hausärztliche Versorgung weniger stark an der Siedlungsstruktur. Dennoch ist nicht in jedem Ort innerhalb ländlicher Regionen eine ärztliche Niederlassung vorhanden. Das bedeutet, dass die Gesundheitsversorgung für Menschen, die in Orten ohne ärztliche Versorgung wohnen, nicht immer fußläufig erreichbar ist. So können in Großstädten weit über $80 \%$ der Einwohner innerhalb von 10 min einen Arzt oder eine Therapieeinrichtung zu Fuß erreichen, in Landge- meinden hingegen nur 62\% [2]. Bei fehlender fußläufiger Erreichbarkeit müssen Verkehrsmittel in Anspruch genommen werden. Vor dem Hintergrund eines erschwerten Zugangs zum öffentlichen Personennahverkehr in ländlichen Räumen nimmt die Abhängigkeit vom Individualverkehr zu [3]. In Deutschland wohnen knapp drei Viertel der Bevölkerung innerhalb eines 10 -minütigen und $97,5 \%$ innerhalb eines 20-minütigen $\mathrm{Pkw}$-Radius zum jeweils nächsten Krankenhaus der Grundversorgung [4].

In einigen Regionen Deutschlands wird sich in den nächsten Jahren die Erreichbarkeit von Einrichtungen der Gesundheitsversorgung voraussichtlich verschlechtern. Vor allem sind davon ländliche Regionen betroffen, da viele Landärzte in den nächsten Jahren aus Altersgründen aus dem Berufsleben ausscheiden werden [5]. Bereits jetzt haben Medizinische Versorgungszentren (MVZ) in diesen Räumen größere Schwierigkeiten, Ärzte zu finden. Im Rahmen des MVZSurveys 2011 der Kassenärztlichen Bundesvereinigung, der regional gegliederte Angaben liefert, äußerten $44 \%$ der befragten MVZ aktuelle Schwierigkeiten durch einen Ärztemangel, in ländlichen Regionen waren es mit 57\% signifikant mehr [6].

Gleichzeitig ist festzustellen, dass aufgrund vieler Studienabbrecher und Studienplatzwechsler die Gesamtzahl an Medizinstudenten fast kontinuierlich sinkt. Zudem entscheiden sich immer weniger
Mediziner für eine Weiterbildung zum Facharzt für Allgemeinmedizin und für eine hausärztliche Tätigkeit, was insbesondere auf geringere Verdienstmöglichkeiten zurückzuführen ist. Vor allem Hausärzte in den neuen Bundesländern haben dementsprechend große Probleme, einen Nachfolger zu finden $[7,8]$.

Infolge der demografischen Alterung wird sich die Anzahl älterer Menschen in Zukunft erhöhen. Damit werden auch altersbedingte gesundheitliche Beschwerden in der Gesellschaft zunehmen. Ältere Menschen weisen im Vergleich zu jüngeren Altersgruppen ein erhöhtes Krankheitsrisiko auf [9]. Hinzu kommt, dass ältere Menschen zumeist an mehreren chronischen Krankheiten (Multimorbidität) erkrankt sind $[9,10]$. Mit zunehmendem Alter wächst die Wahrscheinlichkeit für

- chronische Erkrankungen, die durch das Gesundheitssystem versorgt werden müssen (wie Herzkrankheiten, Neubildungen, Erkrankungen des Muskel-Skelett-Systems, wie beispielsweise Arthrose, Osteoporose usw.),

- Erkrankungen und Einschränkungen, die die individuelle Mobilität beeinträchtigen (wie Herzinsuffizienz, Inkontinenz),

- altersphysiologisch bedingte Einschränkungen (Abbau von Muskelmasse und Muskelkraft, Nachlassen von Gleichwicht, Seh- und Hörkraft) [11]. 

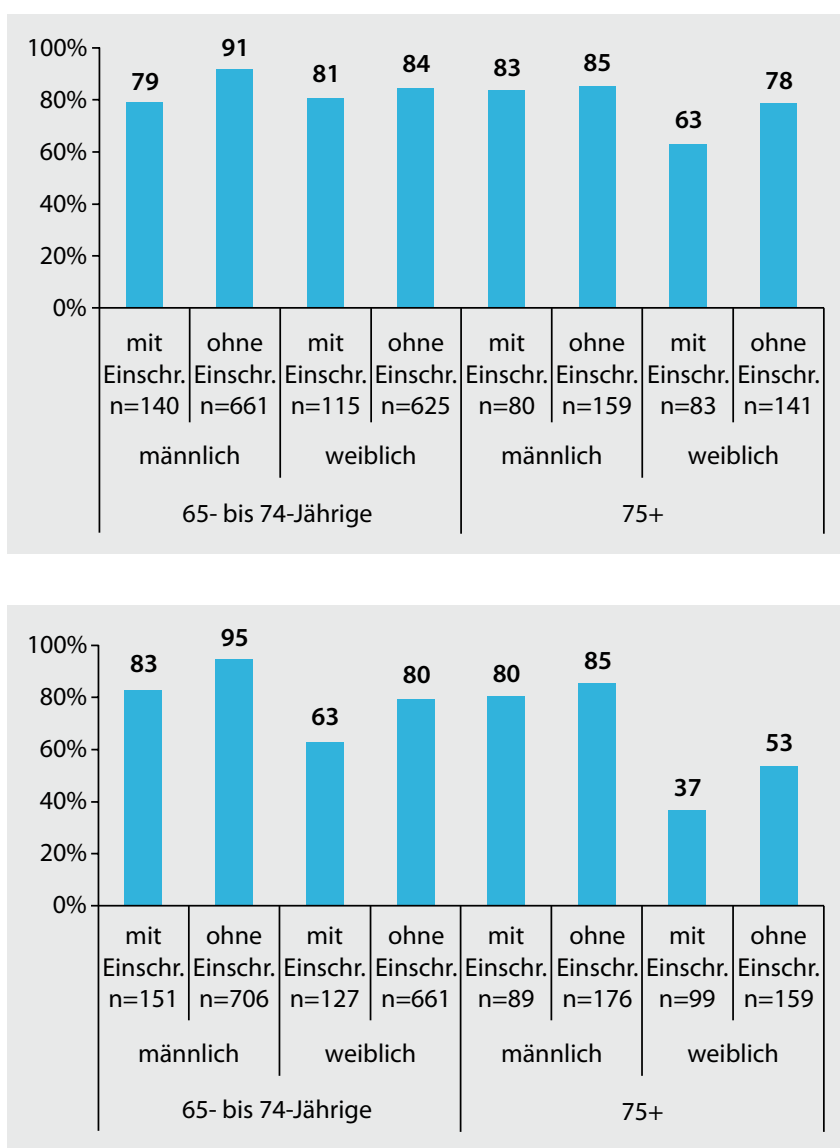

Abb. $1<$ Mobilitätsquote von Älteren in ländlichen Kreisen differenziert nach Geschlecht und Mobilitätseinschränkungen. Eigene Auswertungen auf Basis des Datensatzes "Mobilität in Deutschland 2008". (Nach [17])

Abb. $2<$ Pkw-Verfügbarkeit bei Älteren in ländlichen Kreisen differenziert nach Geschlecht und Mobilitätseinschränkungen. Eigene Auswertungen auf Basis des Datensatzes „Mobilität in Deutschland 2008". (Nach [17])

Ein demografisch bedingter Anstieg der Inanspruchnahme der Gesundheitsversorgung und der Kosten sind die Folge. Die Dynamik dieses Anstiegs kann durch medizinischen Fortschritt, verbesserte Gesundheitsversorgung, Verbesserungen in der gesellschaftlichen individuellen Prävention und Rehabilitation gebremst, aber nicht gänzlich ausgeglichen werden $[12,13]$. Die demografische Alterung weist zudem regionale Differenzierungen auf. In einigen Regionen, vor allem in ländlichen Regionen der neuen Bundesländer, altert die Bevölkerung schneller als in anderen [14].

Damit ergibt sich ein demografisch bedingtes wachsendes Auseinanderklaffen zwischen Nachfragepotenzialen einerseits und Angebotspotenzialen andererseits. Dieses erhält eine besondere Zuspitzung in ländlichen Regionen aufgrund der zu überwindenden räumlichen Entfernungen, die zukünftig tendenziell zunehmen werden. Besonders betroffen hiervon sind ältere Menschen, da ihre individuelle Mobilität überdurchschnittlich häufig aus gesundheitlichen Gründen darüber möglich sein, ob die Gefahr besteht, dass Teile der Älteren nur eingeschränkt an der Gesundheitsversorgung partizipieren können.

\section{Methoden}

Die empirischen Ergebnisse, die im Folgenden ausgeführt werden, basieren auf dem Datensatz der bundesweiten Befragung „Mobilität in Deutschland 2008“ [17]. Mit der wissenschaftlichen Begleitung beauftragte das Bundesministerium für Verkehr, Bau und Stadtentwicklung (BMVBS) das infas Institut für angewandte Sozialwissenschaft $\mathrm{GmbH}$ zusammen mit dem Institut für Verkehrsforschung am Deutschen Zentrum für Luft- und Raumfahrt e.V. (DLR). Im Rahmen dieser Studie wurde von etwa 60.000 Personen in über 25.000 Haushalten in Deutschland das alltägliche Mobilitätsverhalten differenziert erhoben [18].

Der vorliegende Beitrag widmet sich der Alltagsmobilität älterer Menschen in ländlichen Räumen. In Anlehnung an Rosenbaum werden unter Alltagsmobilität „die täglichen Bewegungen von der Wohnung aus zu den Stätten der unterschiedlichsten alltäglichen Aktivitäten“ [19] verstanden. Unberücksichtigt bleiben der Urlaubsverkehr und die residentielle Mobilität, also der Wechsel des Wohnstandortes. Als Raumvariable liegt dieser Analyse der sog. zusammengefasste Kreistyp des Bundesinstituts für Bau-, Stadt- und Raumforschung (BBSR) im Bundesamt für Bauwesen und Raumordnung (BBR) zugrunde, der zwischen Kernstädten, verdichteten Umlandkreisen und ländlichen Kreisen unterscheidet. Als ländliche Kreise, auf die sich die statistischen Analysen nachfolgend beziehen, sind hierbei Kreise bzw. Kreisregionen mit einer Dichte unter 150 Einwohner $/ \mathrm{km}^{2}$ definiert [20].

Die Alltagsmobilität älterer Menschen wird aufgrund der zunehmenden Variabilität des Alters differenziert betrachtet. Neben der Unterscheidung zwischen den ,jungen Alten“ (65 bis 74 Jahre) und den ,alten Alten“ (75 Jahre und älter) erfolgt eine geschlechterdifferenzierte Analyse. Im Kontext dieses Beitrags soll darüber hinaus untersucht werden, welchen Einfluss Mobilitätseinschränkungen für 
die Mobilität älterer Menschen besitzen. Daher wird die betrachtete Gruppe dahingehend unterteilt, ob Einschränkungen in der Mobilität vorhanden sind oder nicht. Im Rahmen der Studie „Mobilität in Deutschland 2008“ wurden die Probanden zunächst gefragt, ob gesundheitliche Einschränkungen vorliegen, und in einem nächsten Schritt, ob durch diese auch Einschränkungen in der Mobilität gegeben sind. Im Folgenden gelten nur die älteren Menschen als mobilitätseingeschränkt, die die Frage nach „Mobilitätseinschränkungen durch gesundheitliche Einschränkung" bejaht haben.

Neben deskriptiven statistischen Analysen und Kontingenzanalysen wird als Auswertungsmethode auch eine binär logistische Regression jeweils unter Verwendung der Statistiksoftware SPSS durchgeführt. $\mathrm{Zu}$ Beginn wird näher auf das Mobilitätsverhalten älterer Menschen in ländlichen Kreisen - differenziert nach den oben genannten Merkmalen - mittels ausgewählter Parameter eingegangen. Im Einzelnen werden Unterschiede bezüglich der Mobilitätsquote, der Pkw-Verfügbarkeit und anhand des Modal Splits herausgestellt. Die Mobilitätsquote beschreibt den Anteil der Personen, die an dem jeweiligen Stichtag der Befragung mobil waren. Mobil waren die Personen, die Wege außerhalb der Wohnung unternommen haben. Die Pkw-Verfügbarkeit gibt an, ob die befragte Person am Stichtag über einen Pkw (als Fahrer oder als Mitfahrer) verfügen konnte. Unter dem Modal Split ist die Aufteilung der Verkehrsmenge auf verschiedene Verkehrsmittel, also die Verkehrsmittelwahl, zu verstehen.

In einem weiteren Schritt wird mithilfe einer binär logistischen Regression untersucht, welche soziodemografischen und raumstrukturellen Einflussfaktoren aus dem vorliegenden Datensatz die Mobilitätsquote älterer Frauen (65 Jahre und älter) in ländlichen Kreisen beeinflussen. Dadurch werden nicht nur die fördernden Rahmenbedingungen für Mobilität aufgezeigt, sondern es wird auch deutlich, welche Teilgruppen aufgrund bestimmter Faktoren in ihrer Mobilität als benachteiligt gelten können. Der Fokus auf ältere Frauen resultiert daraus, dass diese Gruppe im Vergleich zu gleichalt-

Bundesgesundheitsbl 2013 · 56:1418-1424

c) Springer-Verlag Berlin Heidelberg 2013

\section{F. Giesel·K. Köhler · E. Nowossadeck}

Alt und immobil auf dem Land? Mobilitätseinschränkungen älterer Menschen vor dem Hintergrund einer zunehmend problematischen Gesundheitsversorgung in ländlichen Regionen

\section{Zusammenfassung}

Der ländliche Raum ist nicht nur von starken Alterungsprozessen der Bevölkerung gekennzeichnet, sondern auch von einer Gesundheitsinfrastruktur, deren Erreichbarkeit sich voraussichtlich verschlechtern wird. In diesem Kontext stellt sich die Frage, ob ältere Menschen in der Lage sind, die sich vergrößernden räumlichen Distanzen zu überwinden, um bedarfsgerecht medizinisch versorgt zu werden. Demnach ist es das Ziel des vorliegenden Beitrages, die Alltagsmobilität älterer Menschen in ländlichen Räumen differenziert in den Blick zu nehmen. Die empirischen Ergebnisse basieren auf dem Datensatz der bundesweiten Befragung „Mobilität in Deutschland 2008". Die Befunde zeigen, dass besonders ältere Frauen in ihren Mobilitätsoptionen limitiert sind. Mobilitätseingeschränkte Frauen (75+) waren nur zu 63\% außerhäusig mobil und verfügen nur zu 37\% über einen Pkw. Die Verfügbarkeit eines privaten Pkws ist jedoch entscheidend für die außerhäusige Mobilität. Hieraus folgt, dass speziell hochaltrige, mobilitätseingeschränkte Frauen ihren Alltag unter erschwerten Bedingungen bewältigen müssen. Vor dem Hintergrund einer zunehmend problematisch werdenden medizinischen Versorgung in ländlichen Räumen besteht die Herausforderung darin, eine adäquate Grundversorgung für einen großen Anteil immobiler Älterer zu gewährleisten.

Schlüsselwörter Mobilität · Ältere Menschen . Gesundheitsversorgung · Demografischer Wandel · Ländlicher Raum

\section{Old and immobile in rural areas? Limited mobility of the elderly in the context of increasingly problematic health care in rural regions}

\section{Abstract}

Against the background of considerable population ageing processes in rural areas, it is expected that access to the health care system in these regions will deteriorate. Within this context, the question arises as to whether elderly people have the ability to overcome increasing distances in order to receive adequate medical care. Hence the objective of this study is an in-depth analysis of the everyday mobility of elderly people living in rural areas. The empirical analysis is based on the German National Travel Survey "Mobilität in Deutschland 2008". Findings show that older women in particular experience limitations in their mobility options. Only $63 \%$ of mobil- ity-impaired women (aged $>75$ years) were mobile outside their homes, and only $37 \%$ had access to a car, which can be regarded as the crucial factor for being mobile. It follows that older and mobility-impaired women have to cope with everyday life under difficult conditions. In the context of the growing problem of access to the health care system in rural areas, the challenge is to ensure adequate primary health care for a large proportion of the immobile elderly.

Keywords

Travel behaviour $\cdot$ Elderly people $\cdot$ Health care - Demographic change - Rural area rigen Männern - wie noch nachfolgend gezeigt wird - durch geringere Mobilitätsoptionen gekennzeichnet und damit verstärkt gefährdet ist, erhöhte Distanzen nicht überwinden zu können.

\section{Ergebnisse}

Das Mobilitätsverhalten älterer Menschen in ländlichen Kreisen wird zunächst anhand bestimmter Parameter analysiert, die im Folgenden näher ausgeführt werden.

\section{Mobilitätsquote}

- Abb. 1 stellt die Mobilitätsquote von Älteren in ländlichen Kreisen differenziert nach Geschlecht und Mobilitätseinschränkungen dar. Es wird deutlich, dass in den beiden betrachteten Altersgruppen Personen ohne Mobilitätsein- 


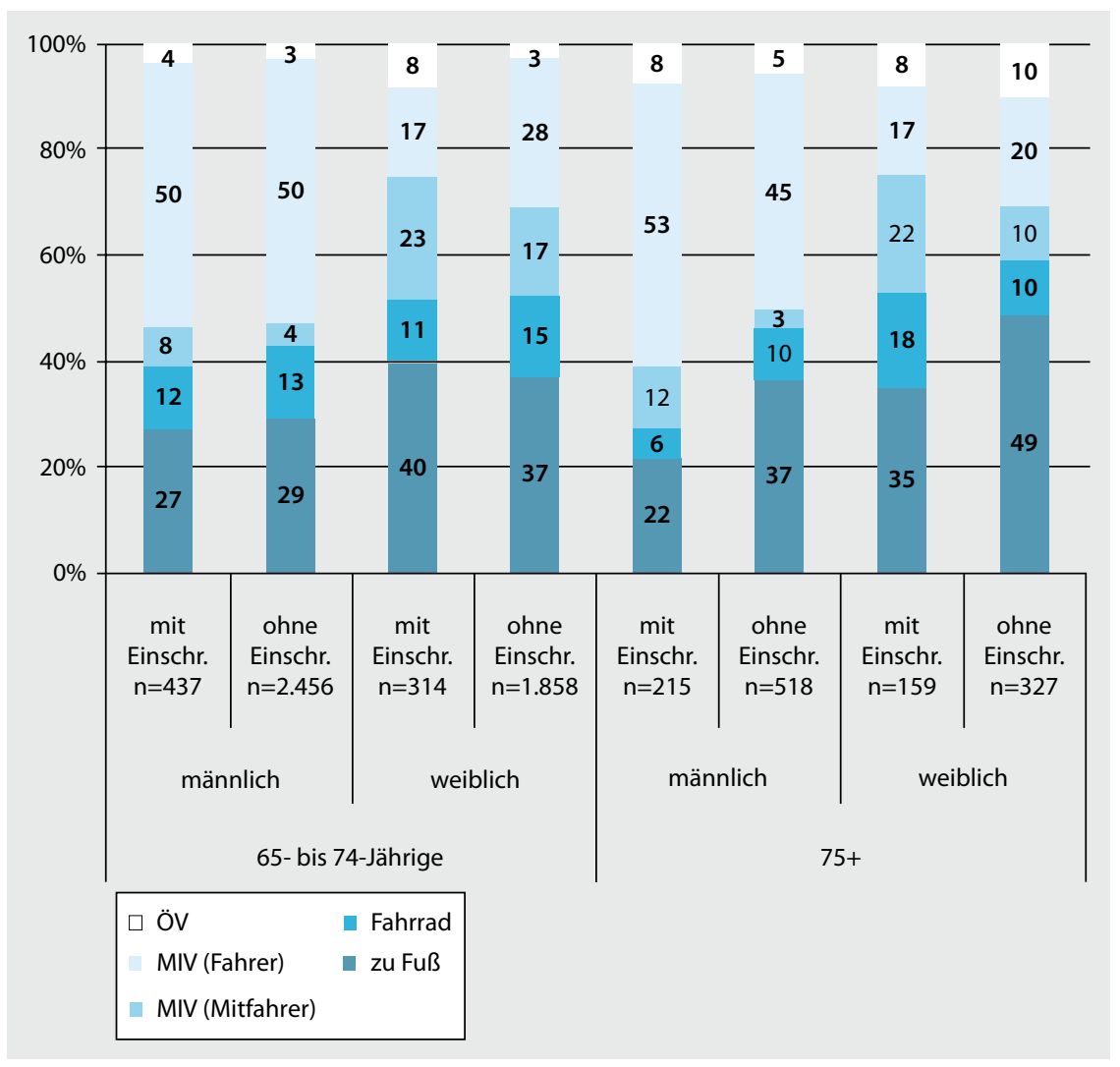

Abb. $3 \Delta$ Modal Split bei Älteren in ländlichen Kreisen differenziert nach Geschlecht und Mobilitätseinschränkungen. Eigene Auswertungen auf Basis des Datensatzes „Mobilität in Deutschland 2008“ [17]. ÖV öffentlicher Verkehr, MIV motorisierter Individualverkehr

schränkungen stets eine höhere Mobilitätsquote aufweisen als mobilitätseingeschränkte Personen gleichen Alters und Geschlechts. Abgesehen von den über 74-jährigen Männern mit Einschränkungen in der Mobilität ist die Mobilitätsquote im höheren Alter im Vergleich zur jüngeren Altersgruppe durchgehend geringer. Zudem ist die Mobilitätsquote der mobilitätseingeschränkten älteren Frauen (75+) auffallend niedrig. So haben in dieser Gruppe am Stichtag nur 63\% Wege außer Haus zurückgelegt, während die mobilitätseingeschränkten Männer derselben Altersgruppe zu 83\% mobil waren.

\section{Pkw-Verfügbarkeit}

Alters- und geschlechtsbedingte Unterschiede können auch bei der Pkw-Verfügbarkeit ausgemacht werden (• Abb. 2). Ältere mit Einschränkungen in ihrer Mobilität konnten seltener auf einen Pkw zurückgreifen als Personen ohne Mobilitätseinschränkungen. Dies gilt auch für Frauen im Vergleich zur jeweiligen männlichen Gruppe. Auch zwischen den Altersgruppen fällt auf, dass sich bei den 65- bis 74-Jährigen durchgehend eine höhere Pkw-Verfügbarkeitsrate zeigt als bei der komplementären Gruppe der über 74-Jährigen. Außer bei den 65- bis 74-Jährigen Frauen ohne Einschränkungen in ihrer Mobilität weicht die Pkw-Verfügbarkeit bei Frauen stark von der bei den Männern ab. Verfügen stets über $80 \%$ der Männer im Alter über einen $\mathrm{Pkw}$, so sind es bei den gleichaltrigen Frauen weitaus weniger. Frauen mit Mobilitätseinschränkungen können sogar nur zu 37\% auf einen Pkw zurückgreifen.

\section{Modal Split}

Die unterschiedlich ausgeprägte PkwVerfügbarkeit zwischen den Altersgruppen und Geschlechtern spiegelt sich auch in der Verkehrsmittelwahl wider (• Abb. 3). Es ist zu erkennen, dass bei den Männern in beiden betrachteten Altersgruppen der motorisierte Individualverkehr (MIV), also insbesonde- re der Pkw, das am häufigsten genutzte Verkehrsmittel ist. Ungefähr jeder zweite Weg wird in der Regel mit einem Pkw zurückgelegt. Öffentliche Verkehrsmittel werden in ländlichen Kreisen kaum genutzt, und Männer sind insgesamt selten als Mitfahrer unterwegs. Mobilitätseinschränkungen wirken sich erst in der Altersgruppe 75+ aus. Männer ohne Mobilitätseinschränkungen gehen häufiger zu Fuß und nutzen häufiger das Fahrrad als Männer mit Einschränkungen in ihrer Mobilität.

Bei Frauen ist die Verkehrsmittelwahl hingegen anders ausgeprägt. Die Verkehrsmittel des motorisierten Individualverkehrs werden weitaus weniger genutzt. Die meisten Wege werden insgesamt zu Fuß zurückgelegt. Die geringe MIV-Nutzung wird teilweise durch den höheren Mitfahreranteil aufgefangen. Bemerkenswert ist auch hier, dass öffentliche Verkehrsmittel nur zu einem geringen Anteil genutzt werden. Im höheren Alter (75+) sticht bei den Frauen, die nicht in ihrer Mobilität eingeschränkt sind, die Dominanz des zu Fuß Gehens hervor. Fast die Hälfte aller Wege wird zu Fuß zurückgelegt und 20\% der Wege immer noch mit dem MIV als Fahrer. Die mobilitätseingeschränkten Frauen derselben Altersgruppe sind hingegen etwas seltener MIV-Fahrer, jedoch sind sie zu einem beachtlichen Anteil (22\%) als Mitfahrer unterwegs. Zu $18 \%$ wird sogar das Fahrrad genutzt, was den größten Anteil der Fahrradnutzung in allen betrachteten Gruppen ausmacht.

Zusammengefasst wird deutlich, dass nicht nur alters- und geschlechtsbedingte Unterschiede hinsichtlich der Mobilität älterer Menschen (65+) ausgemacht werden können, sondern auch, dass Mobilitätseinschränkungen die Mobilitätsquote und die Verkehrsmittelverfügbarkeit und -wahl beeinflussen.

\section{Einflussfaktoren auf die Mobilitätsquote}

Nachfolgend wurde mithilfe einer binär logistischen Regression untersucht, welche soziodemografischen und raumstrukturellen Faktoren aus dem vorliegenden Datensatz die Mobilitätsquote speziell älterer Frauen (65+) in ländlichen 
Tab. 1 Einflussfaktoren auf die Mobilitätsquote älterer Frauen (65+) in ländlichen Kreisen ermittelt mithilfe einer binär logistischen Regression. Eigene Auswertungen auf Basis des Datensatzes „Mobilität in Deutschland 2008“. (Nach [17])

\begin{tabular}{|c|c|c|c|c|c|c|c|}
\hline & Referenzkategorie & Regressionskoeffizient B & Standardfehler & Wald & df & Sig. & $\operatorname{Exp}(B)$ \\
\hline Alter & & $-0,041$ & 0,013 & 10,112 & 1 & 0,001 & 0,960 \\
\hline Pkw-Verfügbarkeit am Stichtag (ja/nein) & Nein & 1,333 & 0,180 & 54,799 & 1 & 0,000 & 3,793 \\
\hline Abitur (ja/nein) & Nein & 1,072 & 0,363 & 8,714 & 1 & 0,003 & 2,922 \\
\hline Wetter (trocken/nass) & Nass & 0,675 & 0,188 & 12,917 & 1 & 0,000 & 1,963 \\
\hline $\begin{array}{l}\text { Erreichbarkeit der Läden zu Fuß } \\
\text { (gut/schlecht) }\end{array}$ & Schlecht & 0,929 & 0,181 & 26,302 & 1 & 0,000 & 2,533 \\
\hline Konstante & & 2,327 & 1,008 & 5,326 & 1 & 0,021 & 10,247 \\
\hline
\end{tabular}

Kreisen beeinflussen. Die Ergebnisse des Modells sind in der $\bullet$ Tab. 1 dargestellt. Die Analyse beruht auf einer gewichteten Fallzahl von 1020 Personen und weist eine Modellgüte von 0,146 (Pseudo-RQuadrat nach McFadden) auf.

Das Modell zeigt, dass die Mobilitätsquote älterer Frauen (65+) in ländlichen Kreisen in erster Linie davon abhängt, $o b$ ein $\mathrm{Pkw}$ verfügbar ist $(\mathrm{p}=0,000)$. Einen positiven Einfluss haben außerdem eine höhere Schulbildung $(p=0,003)$, eine gute fußläufige Erreichbarkeit von Läden und Geschäften des täglichen Bedarfs $(p=0,000)$, eine trockene Wetterlage $(p=0,000)$ und ein geringeres Alter $(\mathrm{p}=0,001)$.

Bezüglich der Variablen Haushaltsgröße sowie der Entfernung zur nächstgelegenen Haltestelle des öffentlichen Personennahverkehrs konnte kein Zusammenhang zur Mobilitätsquote festgestellt werden, sodass diese Variablen nicht im Modell berücksichtigt werden. In einer bivariaten Betrachtung zeigt sich, dass ein signifikanter Zusammenhang ( $\mathrm{p}=0,000)$ zwischen der Pkw-Verfügbarkeit älterer Frauen und dem Führerschein- (Phi $=0,444)$, Fahrradbesitz (Phi $=0,182)$ und dem Vorhandensein von Mobilitätseinschränkungen (Phi $=-0,229)$ besteht. Aufgrund dieses hohen Zusammenhangs mit der Pkw-Verfügbarkeit finden diese Variablen keinen Eingang in das multivariate Modell und werden demnach über die Pkw-Verfügbarkeit miterklärt.

\section{Diskussion}

Die vorliegenden empirischen Ergebnisse verdeutlichen, dass sich die Mobilität älterer Menschen in ländlichen Kreisen abhängig vom Alter, Geschlecht und von Mobilitätseinschränkungen voneinander unterscheidet. Die Unterschiede zwischen den Geschlechtern bestehen hinsichtlich der Mobilitätsquote und der Pkw-Verfügbarkeit bereits in der Altersgruppe der 65- bis 74-Jährigen, diese sind jedoch bei den über 74-Jährigen weitaus stärker ausgeprägt. Frauen in dieser Altersgruppe waren im Vergleich seltener am Stichtag außer Haus und verfügen weitaus seltener über einen Pkw als Männer der gleichen Altersgruppe. Die Mobilitätsquote sowie die Pkw-Verfügbarkeit sind noch geringer, wenn körperlich bedingte Mobilitätseinschränkungen vorliegen. Mobilitätseingeschränkte Frauen im Alter von über 74 Jahren waren nur zu $63 \%$ außerhäusig mobil und verfügen nur zu 37\% über einen Pkw. Diese Ergebnisse sind auf ein Ursachenbündel zurückzuführen.

Die funktionale Gesundheit - also die gesundheitlichen Voraussetzungen für die Bewältigung von Alltagsanforderungen und die Teilnahme am gesellschaftlichen Leben [21] - im Alter hat sich hinsichtlich Hilfebedürftigkeit und Krankheitsprävalenz in den letzten Jahren verbessert [22, 23]. Aufgrund dieser Entwicklung sind von einer erschwerten selbstständigen Lebensführung infolge von gesundheitsbedingten Einschränkungen - wie beispielsweise Einschränkungen beim Treppensteigen - vermehrt erst Menschen in der hochaltrigen Lebensphase betroffen. Hierbei ist jedoch zu beachten, dass körperliche Einschränkungen abhängig vom Geschlecht variieren. Bei älteren Frauen finden sich höhere Prävalenzen für nichtletale Krankheiten, und sie leiden häufiger an Multimorbidität als gleichaltrige Männer [9, 24, 25]. Diese Befunde verdeutlichen, war- um die Mobilitätsquote zwischen den beiden betrachteten Altersgruppen sowie zwischen den Geschlechtern variiert. Die Mobilitätsquote älterer Frauen (75+) ist aber auch auf die geringe $\mathrm{Pkw}$-Verfügbarkeitsrate zurückzuführen, die eng mit ihrer geringen Führerscheinbesitzquote zusammenhängt.

Wenngleich die Führerscheinbesitzquoten bei älteren Menschen (65+) insgesamt zunehmen, bestehen gegenwärtig dennoch große Unterschiede zwischen den Geschlechtern, vor allem bei den Hochaltrigen (75+). Während im Jahr 2008 fast 90\% der älteren Männer (75+) einen Führerschein besaßen, waren es bei Frauen in dieser Altersgruppe nur knapp über 40\% [18]. Der Führerscheinbesitz unterscheidet sich aber auch regional. Historisch bedingt haben ältere Frauen in Ostdeutschland weitaus seltener eine Fahrerlaubnis als westdeutsche Frauen [26].

Die geringe Pkw-Verfügbarkeitsrate wird aber auch von der Haushaltsstruktur beeinflusst. Im Jahr 2009 lebten 44\% der älteren Frauen (65+) in Deutschland in Single-Haushalten, aber nur $18 \%$ der Männer des gleichen Alters. Mit zunehmendem Alter nimmt der Anteil an Single-Haushalten weiter zu. Bei den über 85-Jährigen lebten im Jahr 2009 nur 35\% der Männer allein, aber 74\% der Frauen [27]. Dies ist zum einen auf die höhere Lebenserwartung von Frauen zurückzuführen und zum anderen darin begründet, dass die Mehrheit der Männer mit jüngeren Frauen verheiratet ist. Demnach ist die geringe Pkw-Verfügbarkeit bei älteren Frauen (75+) ein Resultat geringer Pkw-Führerscheinbesitzquoten sowie des wegfallenden Pkws im Haushalt nach dem Tod des Mannes [28]. 
Hinsichtlich des Modal Splits können auch Differenzen festgestellt werden. Bei den Männern im Alter von 65 bis 74 Jahren unterscheidet sich die Verkehrsmittelwahl jedoch nicht darin, ob körperliche Einschränkungen gegeben sind. Jeder zweite Weg wird mit dem motorisierten Individualverkehr zurückgelegt und fast ein Drittel zu Fuß. Die Bedeutung des MIVs bleibt auch im Alter aufgrund hoher Führerscheinbesitzquoten konstant. Öffentliche Verkehrsmittel sowie das Fahrrad spielen eine untergeordnete Rolle. Bei den Frauen in dieser Altersgruppe werden die meisten Wege zu Fuß getätigt. In der Mobilität eingeschränkte Frauen nutzen für fast ein Viertel der Wege den privaten Pkw. Dieser Wert ist bei den nicht mobilitätseingeschränkten Personen mit 6 Prozentpunkten geringer, wobei hier der Mitfahreranteil höher ist. Hieran ist zu erkennen, dass mobilitätseingeschränkte Frauen vermehrt auf die Nutzung des motorisierten Individualverkehrs angewiesen sind. Bei den über 74-Jährigen Frauen mit Einschränkungen in der Mobilität bleibt der Modal Split annähernd konstant. Bemerkenswerterweise ist die Nutzung des MIVs bei den nicht eingeschränkten älteren Frauen im Vergleich geringer, obwohl eine höhere Pkw-Verfügbarkeitsrate vorliegt. Fast die Hälfte der Wege wird zu Fuß zurückgelegt. Dies ist wiederum ein Indiz dafür, dass mobilitätseingeschränkte Frauen längere Wege zurücklegen müssen und dabei nicht auf die Nutzung des motorisierten Individualverkehrs verzichten können. Neben der selbstständigen Nutzung ist bei den mobilitätseingeschränkten älteren Frauen auch die Möglichkeit des Mitfahrens von Bedeutung. Hervorzuheben ist hierbei noch, dass öffentliche Verkehrsmittel fast keine Rolle für die Alltagsmobilität älterer Menschen in ländlichen Kreisen spielen, was auf eine fehlende, nicht alters- oder bedarfsgerechte Infrastruktur hinweisen kann.

Anhand der Ergebnisse der binär logistischen Regression können förderliche Rahmenbedingungen für die individuelle Mobilität - gemessen an der Mobilitätsquote - identifiziert werden. Es wird deutlich, dass die Mobilität älterer Frauen entscheidend von der Pkw-Verfügbarkeit abhängt. Als weitere bestimmen- de Faktoren konnten die Schulbildung, die fußläufige Erreichbarkeit von Läden und Geschäften des täglichen Bedarfs, die Wetterlage sowie das Alter identifiziert werden. Der Einfluss von Mobilitätseinschränkungen wird aufgrund der hohen Korrelation mit der Pkw-Verfügbarkeit über diese Variable miterklärt, sodass die Analyseergebnisse nicht belegen, dass gesundheitlich bedingte Einschränkungen keinen Einfluss auf die Mobilität haben. Zusammengefasst besteht demnach gegenwärtig die Gefahr, dass hochaltrige mobilitätseingeschränkte Frauen ohne Pkw-Verfügbarkeit in ihren Mobilitätsoptionen limitiert sind und u. a. an Gesundheitsdienstleistungen in ländlichen Kreisen nur sehr erschwert partizipieren können. Insbesondere sozial benachteiligte ältere Frauen in monofunktionalen Wohnumfeldern sind hierbei auf Unterstützung angewiesen.

Eine schlechtere Erreichbarkeit der ambulanten Versorgung kann ihre sinkende Inanspruchnahme und damit einen sich verschlechternden Gesundheitszustand nach sich ziehen. Es ist gezeigt worden, dass eine niedrige Ärztedichte und eine hohe Zahl an vermeidbaren Krankenhausfällen miteinander korrelieren [29]. Neben negativen Auswirkungen auf individueller Ebene ist also mit ungünstigen Folgen für die Gesundheitskosten zu rechnen, denn Krankenhausaufenthalte sind teurer als ambulante Arztbesuche.

Der Problemlage kann auf 2 Wegen begegnet werden. Einerseits müssen die Potenziale mittels innovativer Konzepte ausgeschöpft werden, um die Mobilität älterer Menschen ohne Pkw-Verfügbarkeit generell zu erhöhen. Hier ist nicht nur ein leistungsfähiger öffentlicher Personennahverkehr von Bedeutung, der alters- und geschlechtsspezifischen Mobilitätsbedürfnissen gerecht wird, sondern es ist auch der Einsatz von Carsharing oder von Elektromobilität in diesen räumlichen Kontexten anzudenken. Darüber hinaus ist Eigenverantwortung in Form zivilgesellschaftlicher Initiativen unbedingt notwendig. Nachbarschaftliche Netzwerke und Freiwilligendienste vermögen beispielsweise über die Einrichtung von Bürgerbussen oder Fahrgemeinschaften zu einer Verbesserung der Mobilität ins- besondere zunehmend immobiler Personen in dünn besiedelten Regionen beizutragen.

Andererseits erfordert die dargestellte Situation neue Versorgungskonzepte, die teilweise in den letzten Jahren erprobt wurden oder bereits Versorgungsrealität sind. Hier seien nur 3 Beispiele benannt:

- Delegierung ärztlicher Tätigkeiten auf qualifizierte nichtärztliche Fachkräfte („AGnES“ [30], „MoNi“ [31], „VERAH“ und weitere Modellprojekte [32]), die die Patienten zu Hause aufsuchen und sie entsprechend ihrem Auftrag betreuen und damit die Hausärzte in ländlichen unterversorgten Regionen entlasten,

- Etablierung eines sog. „Patientenbusses“, der Patienten von ihrem Wohnort abholt und in die Nähe der Arztpraxen bringt [33],

- Nutzung moderner Informationsund Kommunikationstechnologien in Kombination mit diagnostischen und therapeutischen Maßnahmen. Eine Auswahl verschiedener telemedizinischer Methoden findet sich in [34]. Ziel dieser verschiedenen Projekte ist es u. a., die Reichweite ärztlichen Handelns zu verlängern, um regionale Versorgungsunterschiede auszugleichen [35].

\section{Fazit}

Die vorliegende Analyse hat die enorme Bedeutung des Pkws in ländlichen Kreisen für ältere Menschen aufgezeigt. Ist eine selbstständige Nutzung des Pkws aus medizinischen Gründen oder aufgrund einer fehlenden Fahrerlaubnis nicht möglich, so sind ältere Menschen als Mitfahrer auf den Pkw des Partners oder auf einen Pkw im nachbarschaftlichen oder familialen Netzwerk angewiesen. Insbesondere hochaltrige alleinlebende Frauen, die von gesundheitlichen Einschränkungen betroffen sind, stehen vor der Herausforderung, ihren Alltag - vor allem unerlässliche längere Wege zu Ärzten - unter erschwerten sozialen und räumlichen Bedingungen zu bewältigen. Aus der eingangs skizzierten tendenziell negativen Entwicklung der ambulanten und stationären medizinischen Grundversorgung speziell in ländlichen 
Räumen resultiert das Problem, zukünftig eine adäquate Grundversorgung für einen zunehmenden Anteil immobiler Älterer zu gewährleisten.

\section{Korrespondenzadresse}

\section{F. Giesel}

Institut für Verkehrsforschung,

Deutsches Zentrum für Luft- und Raumfahrt

e.V. (DLR)

Rutherfordstr. 2, 12489 Berlin

Flemming.Giesel@dlr.de

Interessenkonflikt. F. Giesel, K. Köhler und E. Nowossadeck geben an, dass kein Interessenkonflikt besteht.

Dieser Beitrag beinhaltet keine Studien an Menschen oder Tieren.

\section{Literatur}

1. BMVBS, Bundesministerium für Verkehr, Bau und Stadtentwicklung (Hrsg) (2012) Mobilität, Erreichbarkeit und soziale Exklusion. Fähigkeiten und Ressourcen einer ländlichen Bevölkerung für eine angemessene Versorgung und Teilhabe am öffentlichen Leben. BMVBS-Online-Publikation 27/2012

2. Sturm G, Walther A (2011) Lebensqualität in kleinen Städten und Landgemeinden. Aktuelle Befunde der BBSR-Umfrage. BBSR-Berichte kompakt, 5. Bundesinstitut für Bau-, Stadt- und Raumforschung (BBSR) im Bundesamt für Bauwesen und Raumordnung (BBR), Bonn

3. Greß S, Stegmüller K (2011) Gesundheitliche Versorgung in Stadt und Land - Ein Zukunftskonzept. Expertise für die Friedrich-Ebert-Stiftung. Friedrich-Ebert-Stiftung, Wiesbaden

4. Schlömer C, PützT (2011) Bildung, Gesundheit, Pflege - Auswirkungen des demographischen Wandels auf die soziale Infrastruktur. BBSR-Berichte Kompakt 11:1-16

5. KBV, Kassenärztliche Bundesvereinigung (2011) Grunddaten zur vertragsärztlichen Versorgung in Deutschland 2011. http://www.kbv.de/publikationen/125.html (Zugegriffen: 04.04.2013)

6. KBV, Kassenärztliche Bundesvereinigung (2012) MVZ-Survey 2011. Medizinische Versorgungszentren in ländlichen Regionen, Berlin

7. Kopetsch T (2010) Dem deutschen Gesundheitswesen gehen die Ärzte aus! Studie zur Altersstruktur- und Arztzahlenentwicklung, 5. aktualisierte und komplett überarbeitete Aufl. Bundesärztekammer und Kassenärztliche Bundesvereinigung, Berlin

8. Gerlinger T (2011) Versorgung in ländlichen Regionen. Public Health Forum 70(19):13.e1-13.e3

9. Fuchs J, Busch M, Lange C, Scheidt-Nave C (2012) Prevalence and patterns of morbidity among adults in Germany. Results of the German telephone health Interview survey German Health Update (GEDA) 2009. Bundesgesundheitsbl Gesundheitsforsch Gesundheitsschutz 55:576-586

10. Nowossadeck S, Nowossadeck E (2011) Krankheitsspektrum und Sterblichkeit im Alter. Report Altersdaten 1-2/2011. Deutsches Zentrum für Altersfragen, Berlin
11. Böhm K, Tesch-Römer C, Ziese T (Hrsg) (2009) Gesundheit und Krankheit im Alter. Beiträge zur Gesundheitsberichterstattung des Bundes. Robert Koch-Institut, Berlin

12. Nowossadeck E (2010) Morbiditätsprognosen auf Basis von Bevölkerungsprognosen. Welchen Beitrag kann ein Gesundheitsmonitoring leisten? Bundesgesundheitsbl Gesundheitsforsch Gesundheitsschutz 53:427-434

13. Adler G, Knesebeck J-H v.d. (2011) Ärztemangel und Ärztebedarf in Deutschland. Bundesgesundheitsbl Gesundheitsforsch Gesundheitsschutz 54:228-237

14. Menning S, Nowossadeck E, Maretzke S (2010) Regionale Aspekte der demografischen Alterung. Report Altersdaten 1-2/2010. Deutsches Zentrum für Altersfragen, Berlin

15. Pohl T, Giesel F, Oßenbrügge J (2010) Suburbia als „räumliche Falle"? - Folgen der demographischen und sozialräumlichen Entwicklung von Großstädten am Beispiel Hamburgs. Berichte zur deutschen Landeskunde 4:329-348

16. Giesel F, Jarass J (2013) Nahräumliche Mobilität im Alter? Mobilitätsverhalten älterer Menschen in Berliner Sozialräumen. Österr Z Verkehrswissenschaft 1-2:20-33

17. infas, DLR (2010) Mobilität in Deutschland 2008. Basisdatensatz. Untersuchung im Auftrag des Bundesministeriums für Verkehr, Bau und Stadtentwicklung (BMVBS), Bonn. http://www.clearingstelle-verkehr.de

18. infas, DLR (2010) Mobilität in Deutschland 2008. Ergebnisbericht: Struktur - Aufkommen - Emissionen - Trends. Untersuchung im Auftrag des Bundesministeriums für Verkehr, Bau und Stadtentwicklung (BMVBS), Bonn

19. Rosenbaum W (2007) Mobilität im Alltag - Alltagsmobilität. In: Schöller O, Canzler W, Knie A (Hrsg) Handbuch Verkehrspolitik. VS Verlag für Sozialwissenschaften, Wiesbaden, S 549-572

20. BBSR, Bundesinstitut für Bau-, Stadt- und Raumforschung (2013) Raumbeobachtung. http://www. raumbeobachtung.de

21. Menning S, Hoffmann E (2009) Funktionale Gesundheit und Pflegebedürftigkeit. In: Böhm K, Tesch-Römer C, Ziese T (Hrsg) Gesundheit und Krankheit im Alter. Beiträge zur Gesundheitsberichterstattung des Bundes. Robert Koch-Institut, Berlin, S 62-78

22. Dinkel R (1999) Demographische Entwicklung und Gesundheitszustand. Eine empirische Kalkulation der Healthy Life Expectancy für die Bundesrepublik auf der Basis von Kohortendaten. In: Häfner H (Hrsg) Gesundheit - unser höchstes Gut? Springer, Berlin, S 61-82

23. Freedman VA, Marin LG, Schoeni RF (2002) Recent trends in disability and functioning among older adults in the United States. A systematic review. J Am Med Assoc 288(24):3137-3146

24. Ahacic K, Parker MG, Thorslund M (2007) Aging in disguise: age, period and cohort effects in mobility and edentulousness over three decades. Eur J Ageing 4:83-91

25. Wurm S, Schöllgen I, Tesch-Römer C (2010) Gesundheit. In: Motel-Klingebiel A, Wurm S, TeschRömer C (Hrsg) Altern im Wandel. Befunde des Deutschen Alterssurveys (DEAS). W. Kohlhammer, Stuttgart, S 90-117

26. infas, DIW (2004) Mobilität in Deutschland 2002. Ergebnisbericht. Untersuchung im Auftrag des Bundesministeriums für Verkehr, Bau- und Wohnungswesen (BMVBW), Bonn
27. Statistisches Bundesamt (Hrsg) (2011) Ältere Menschen in Deutschland und der EU. Statistisches Bundesamt, Wiesbaden

28. Ottmann P (2010) Abbildung demographischer Prozesse in Verkehrsentstehungsmodellen mit Hilfe von Längsschnittdaten. Schriftenreihe des Instituts für Verkehrswesen, Bd. 69. Eigenverlag, Karlsruhe

29. Sundmacher L, Busse R (2012) Der Einfluss der Arztdichte auf ambulant-sensitive Krankenhausfälle. In: Klauber J, Geraedts M, Friedrich J, Wasem J (Hrsg) Krankenhausreport 2012. Schwerpunkt: Regionalität. Schattauer, Stuttgart, S 183-202

30. Berg N van den, Meinke C, Heymann R et al (2009) AGnES: Hausarztunterstützung durch qualifizierte Praxismitarbeiter. Dtsch Arztebl Int 106(1-2):3-9

31. Beneker C (2013) Verlängert. MoNi geht weiter auf Hausbesuch. Ärzte Zeitung, 12.02.2013

32. Kalitzkus V, Schluckebier I, Wilm S (2009) AGnES, EVA, VerAH und Co - Wer kann den Hausarzt unterstützen und wie? Experten diskutieren die Zukunft der Medizinischen Fachangestellten in der hausärztlichen Versorgung. Z Allgemeinmed 85(10):403-405

33. Kassenärztliche Vereinigung Brandenburg (2012) „Patientenbus" wird medizinische Versorgung verbessern. Pressemitteilung der Kassenärztlichen Vereinigung Brandenburg. http://www.kvbb.de/ presse/pressemitteilungen/newsdetail/article/patientenbus-wird-medizinische-versorgung-verbessern/1/ (Zugegriffen: 24.04.2013)

34. Bartmann FJ, Blettner M, Heuschmann PU (Hrsg) (2012) Telemedizinische Methoden in der Patientenversorgung. Anwendungsspektrum, Chancen, Risiken. Deutscher Ärzte-Verlag, Köln

35. Bartmann FJ, Blettner M, Heuschmann PU (2012) Einführung. In: Bartmann FJ, Blettner M, Heuschmann PU (Hrsg) (2012) Telemedizinische Methoden in der Patientenversorgung. Anwendungsspektrum, Chancen, Risiken. Deutscher Ärzte-Verlag, Köln 